\title{
ON SOME SINGULAR MONOTONIC FUNCTIONS WHICH ARE STRICTLY INCREASING
}

\author{
BY \\ R. SALEM
}

1. A continuous non-decreasing function $f(x)$ defined for $0 \leqq x \leqq 1(f(0)=0$, $f(1)=1$ ) and which is purely singular, that is to say, which has the property

$$
d f / d x=0
$$

almost everywhere, may be constant in every interval contiguous to a perfect set of measure zero: it is usually said, in this case, that $f(x)$ is of the Cantor type. There are, however, monotonic continuous functions, purely singular, which are increasing in the strict sense, that is, $f\left(x^{\prime}\right)>f(x)$ whenever $x^{\prime}>x$.

While the existence of functions of the Cantor type is almost intuitive and their construction is immediate by successive approximations, the existence of strictly increasing singular functions lies deeper. Actually, if we except Minkowski's function? $(x)$, of which we shall speak later (and whose singularity is by no means obvious), no simple direct construction of such functions seems to be known. Functions of this type usually have been obtained by "convolutions" of functions of the Cantor type and the proof that they are singular strictly increasing functions is somewhat difficult $\left({ }^{1}\right)$. Thus, it seems to be of interest to give simple direct constructions of strictly increasing singular functions.

2. Let us consider, in the plane, the straight line $P Q$ joining the point $P$ of cartesian coordinates $x, y$, to the point $Q$ of cartesian coordinates $x+\Delta x$, $y+\Delta y, \Delta x>0, \Delta y>0$. Let $\lambda_{0}, \lambda_{1}$ be two numbers, essentially positive, such that $\lambda_{0}+\lambda_{1}=1\left(\lambda_{0} \neq \lambda_{1}\right)$. Let us now consider the point $R$ whose coordinates are

$$
x+\Delta x / 2, \quad y+\lambda_{0} \Delta y,
$$

that is to say, the horizontal distance between $P, R$ or between $Q, R$ is $\Delta x / 2$, while the vertical distance between $P, R$ is $\lambda_{0} \Delta y$, and the vertical distance between $R, Q$ is $\lambda_{1} \Delta y$. If we replace the straight line $P Q$ by the broken line $P R Q$, we will say that we perform on $P Q$ the transformation $T\left(\lambda_{0}, \lambda_{1}\right)$.

Presented to the Society, November 28, 1942; received by the editors October 8, 1942.

(1) See for example Jessen and Wintner, Distribution functions and the Riemann zeta function, Trans. Amer. Math. Soc. vol. 38 (1935) pp. 48-88 and particularly p. 61; Kershner and Wintner, On symmetric Bernoulli convolutions, Amer. J. Math. vol. 57 (1935) pp. 541-548; Wiener and Wintner, Fourier-Stieltjes transforms and singular infinite convolutions, Amer. J. Math. vol. 60 (1938) pp. 513-522 and particularly p. 521. For earlier examples see Denjoy, J. Math.Pures Appl. 1915 pp. 204-209 (which was the first example given); Sierpinski, Giornale di Matematiche vol. 54 (1916) pp. 314-334; Rajchman, Fund. Math. vol. 2 (1921) pp. 50-63. 
Definition of a function $f(x)$. Let now $f_{0}(x)$ be for $0 \leqq x \leqq 1$ the function equal to $x$, that is to say represented by the straight line $O A$ joining the origin $O$ to the point $A(1,1)$. Let us perform on $O A$ the transformation $T\left(\lambda_{0}, \lambda_{1}\right)$. We get a broken line consisting of two straight lines and representing an increasing function $f_{1}(x)$. Let us perform on each of those two straight lines the transformation $T\left(\lambda_{0}, \lambda_{1}\right)$. We get a broken line consisting of $2^{2}$ straight lines and representing an increasing function $f_{2}(x)$. Proceeding in the same way we get after $p$ operations a function $f_{p}(x)$ strictly increasing $\left(f_{p}(0)=0, f_{p}(1)=1\right)$ represented by a polygonal line consisting of $2^{p}$ straight lines, the vertices having for abscissae the points $k / 2^{p}\left(k=1,2, \cdots, 2^{p}-1\right)$.

Putting

$$
\max \left(\lambda_{0}, \lambda_{1}\right)=\mu
$$

we have essentially, by our hypothesis, $\mu<1$, and it is immediately seen that

$$
\left|f_{p+1}-f_{p}\right| \leqq \mu^{p} \text {. }
$$

Thus $f_{p}(x)$ converges uniformly to a continuous function $f(x) \quad(f(0)=0$, $f(1)=1)$. This function $f(x)$ is strictly increasing because for every $p$ the vertices of the curve $y=f_{p}(x)$ belong to the curve $y=f(x)$ : thus if $f(x)$ was constant in some interval, there would be a $p$ for which two different vertices of $y=f_{p}(x)$ would have the same ordinate, which is impossible.

The ordinate of the vertex of $y=f_{p}(x)$ whose abscissa is given by

$$
\theta_{1} / 2+\theta_{2} / 2^{2}+\cdots+\theta_{p} / 2^{p} \quad\left(\theta_{i}=0 \text { or } 1\right)
$$

is given by

$$
\lambda_{0}\left[\theta_{1}+\lambda_{\theta_{1}} \theta_{2}+\lambda_{\theta_{1}} \lambda_{\theta_{2}} \theta_{3}+\cdots+\lambda_{\theta_{1}} \lambda_{\theta_{2}} \cdots \lambda_{\theta_{p-1}} \theta_{p}\right]
$$

and thus, by continuity, if

$$
x=\theta_{1} / 2+\theta_{2} / 2^{2}+\cdots+\theta_{p} / 2^{p}+\cdots,
$$

we have

$$
f(x)=\lambda_{0}\left[\theta_{1}+\lambda_{\theta_{1}} \theta_{2}+\lambda_{\theta_{1}} \lambda_{\theta_{2}} \theta_{3}+\cdots+\lambda_{\theta_{1}} \lambda_{\theta_{2}} \cdots \lambda_{\theta_{p-1}} \theta_{p}+\cdots\right]
$$

the series being obviously convergent. If $x$ has two different dyadic developments, the formula (3) gives for $f(x)$ the same value.

Let us remark also that if $x$ and $x^{\prime}>x$ have the first $p$ digits of their dyadic developments identical, and equal to $\theta_{1}, \theta_{2}, \cdots, \theta_{p}$, then

$$
f\left(x^{\prime}\right)-f(x)<\lambda_{\theta_{1}} \lambda_{\theta_{2}} \cdots \lambda_{\theta_{p}} .
$$

This is seen immediately by the formula (3) or geometrically.

Proof that $f(x)$ is singular. We shall now prove that the function $f(x)$ is singular. 
It is well known that almost all numbers in $(0,1)$ are "normal" in the scale of 2 , that is, are such that

$$
\theta_{1}+\theta_{2}+\cdots+\theta_{p}=p / 2+o(p) \text { when } p \rightarrow \infty .
$$

Let $N$ be the set of these normal numbers. We have meas $N=1$. Let us fix an $x$ belonging to $N$. Let $x$ be given by (2). $p$ being a positive integer the number $x+\epsilon_{p+1} / 2^{p+1}$, where

$$
\begin{array}{lll}
\epsilon_{p+1}=1 & \text { if } & \theta_{p+1}=0 \\
\epsilon_{p+1}=-1 & \text { if } & \theta_{p+1}=1,
\end{array}
$$

has a dyadic development whose first $p$ digits are the same as for $x$, that is, $\theta_{1}, \theta_{2}, \cdots, \theta_{p}$.

Hence by (4)

$$
\left|f\left(x+\epsilon_{p+1} / 2^{p+1}\right)-f(x)\right|<\lambda_{\theta_{1}} \lambda_{\theta_{2}} \cdots \lambda_{\theta_{p}} .
$$

Now $x$ being normal

$$
\theta_{1}+\theta_{2}+\cdots+\theta_{p}=p / 2+\phi(p)
$$

with $|\phi(p)| / p \rightarrow 0$ when $p \rightarrow \infty$. Hence

$$
\lambda_{\theta_{1}} \lambda_{\theta_{2}} \cdots \lambda_{\theta_{p}}=\lambda_{0}^{p / 2-\phi(p)} \lambda_{1}^{p / 2+\phi(p)}<\left(\lambda_{0} \lambda_{1}\right)^{p / 2-|\phi(p)|}
$$

hence

$$
2^{p+1}\left|f\left(x+\epsilon_{p+1} / 2^{p+1}\right)-f(x)\right|<\left(2\left(\lambda_{0} \lambda_{1}\right)^{1 / 2}\right)^{p \cdot 2 /\left(\lambda_{0} \lambda_{1}\right)^{|\phi(p)|} .}
$$

Now $\lambda_{0}$ and $\lambda_{1}$ being essentially different and $\lambda_{0}+\lambda_{1}$ being equal to 1 , we have

$$
2\left(\lambda_{0} \lambda_{1}\right)^{1 / 2}<1 \text {. }
$$

This, together with $\lim |\phi(p)| / p=0$ proves that the second member of (5) tends to zero for $p=\infty$, and thus, if $f(x)$ has a derivative at the point $x$, this derivative cannot have a value different from zero. But by a classical theorem $f^{\prime}(x)$ exists and is finite almost everywhere, hence almost everywhere in $N$. Hence, also almost everywhere, $f^{\prime}(x)=0$ which proves our theorem.

Modulus of continuity of $f(x)$. The vertical distance between two vertices of abscissae $k / 2^{p}, k+1 / 2^{p}$ being less than $\mu^{p}$ where $\mu$ is defined by (1), we have immediately that if $1 / 2^{p+1} \leqq x^{\prime}-x<1 / 2^{p}$ then

$$
f\left(x^{\prime}\right)-f(x)<2 \mu^{p} \leqq 2 \mu^{-1} \mu^{\log \left(x^{\prime}-x\right)^{-1 / \log 2}}=2 \mu^{-1}\left(x^{\prime}-x\right)^{|\log \mu| / \log 2} .
$$

Hence $f(x)$ satisfies a Lipschitz condition of order $|\log \mu| / \log 2$.

Fourier-Stieltjes coefficients of $f(x / 2 \pi)$. Let

$$
c_{n}=\int_{0}^{2 \pi} e^{n i x} d f(x / 2 \pi) .
$$


We divide the $y$-axis by the points of subdivision corresponding to the vertices existing at the pth stage of the construction of the function and we observe that the vertical distance between the vertex whose abscissa is

$$
2 \pi\left(\theta_{1} / 2+\theta_{2} / 2^{2}+\cdots+\theta_{p} / 2^{p}\right)
$$

and the following one, which can be written

$$
2 \pi\left(\theta_{1} / 2+\theta_{2} / 2^{2}+\cdots+\theta_{p} / 2^{p}+\sum_{s=p+1}^{\infty}\left(1 / 2^{s}\right)\right)
$$

has the value $\lambda_{\theta_{1}} \lambda_{\theta_{2}} \cdots \lambda_{\theta_{p}}$. We thus get for approximate expression of the integral

$$
\sum \lambda_{\theta_{1}} \lambda_{\theta_{2}} \cdots \lambda_{\theta_{p}} e^{2 \pi n i\left(\theta_{1} / 2+\theta_{2} / 2^{2}+\cdots+\theta_{p} / 2^{p}\right)},
$$

the summation being extended to the $2^{p}$ combinations of the values 0 and 1 of the $\theta_{i}$. This sum is equal to

$$
\prod_{k=1}^{p}\left[\lambda_{0}+\lambda_{1} e^{2 \pi n i / 2 k}\right]
$$

and thus, making $p=\infty$, we have

$$
c_{n}=\prod_{k=1}^{\infty}\left[\lambda_{0}+\lambda_{1} e^{2 \pi n i / 2 k}\right] .
$$

We can also write

$$
c_{n}=\prod_{k=1}^{\infty} e^{\pi n i / 2 k}\left[\lambda_{0} e^{-\pi n i / 2^{k}}+\lambda_{1} e^{\pi n i / 2^{k}}\right]=e^{\pi n i} \prod_{k=1}^{\infty}\left[\lambda_{0} e^{-\pi n i / 2^{k}}+\lambda_{1} e^{\pi n i / 2^{k}}\right],
$$

or, putting $\lambda_{0}=(1-r) / 2, \lambda_{1}=(1+r) / 2$

that gives

$$
c_{n}=e^{\pi n i} \prod_{k=1}^{\infty}\left[\cos \left(\pi n / 2^{k}\right)+i r \sin \left(\pi n / 2^{k}\right)\right]
$$

$$
\left|c_{n}\right|^{2}=\prod_{k=1}^{\infty}\left[\cos ^{2}\left(\pi n / 2^{k}\right)+r^{2} \sin ^{2}\left(\pi n / 2^{k}\right)\right] .
$$

If we take $n=2^{m}$, we have

$$
\left|c_{2^{m}}\right|^{2}>r^{2} \cos ^{2}(\pi / 4) \cos ^{2}(\pi / 8) \cos ^{2}(\pi / 16) \cdots
$$

and thus $c_{n}$ does not tend to zero for $n=\infty$.

3. Generalization of the preceding function. Instead of constructing our function with an infinity of identical transformations $T\left(\lambda_{0}, \lambda_{1}\right)$, let us change the transformation used at every step of the construction.

Thus $F_{0}(x)$ being equal to $x$ in $(0,1)$ let $O$ be the point $(0,0), A$ the point 
$(1,1)$ and let us perform on $O A$ the transformation $T\left(\lambda_{0}^{(1)}, \lambda_{1}^{(1)}\right)$. We get a broken line consisting of two straight lines and representing $F_{1}(x)$. On each of those two straight lines we perform the transformation $T\left(\lambda_{0}^{(2)}, \lambda_{1}^{(2)}\right)$; on the $2^{2}$ straight lines constituting $F_{2}(x)$ we perform the transformation $T\left(\lambda_{0}^{(3)}, \lambda_{1}^{(3)}\right)$ to get $F_{3}(x)$, and so on.

Let

$$
\lambda_{0}^{(k)}=\left(1-r_{k}\right) / 2, \quad \lambda_{1}^{(k)}=\left(1+r_{k}\right) / 2 .
$$

We assume that $-1<r_{k}<1$ for every $k$ and that if we put

$$
u_{p}=\prod_{k=1}^{p}\left(\frac{1+\left|r_{k}\right|}{2}\right)
$$

the series $\sum u_{p}$ converges. (This is certainly the case, for example, if $-\alpha<r_{k}<\alpha$, $0<\alpha<1$, but can be secured under less stringent conditions.) Then there is no change in the argument used in $\$ 2$ to prove that $F_{p}(x)$ tends uniformly to a continuous function $F(x)$ strictly increasing from 0 to 1 in the interval $(0,1)$.

In the same way as before we prove that if

$$
x=\theta_{1} / 2+\theta_{2} / 2^{2}+\cdots+\theta_{p} / 2^{p}+\cdots,
$$

we have

$$
F(x)=\theta_{1} \lambda_{0}^{(1)}+\theta_{2} \lambda_{\theta_{1}}^{(1)} \lambda_{0}^{(2)}+\theta_{3} \lambda_{\theta_{1}}^{(1)} \theta_{\theta_{2}}^{(2)} \lambda_{0}^{(3)}+\cdots \cdot
$$

Finally, if $x$ and $x^{\prime}>x$ have the same first $p$ digits in their dyadic development, we have

$$
F\left(x^{\prime}\right)-F(x)<\lambda_{\theta_{1}}^{(1)} \lambda_{\theta_{2}}^{(2)} \cdots \lambda_{\theta_{p}}^{(p)}=\left(1 / \dot{2}^{p}\right) \prod_{k=1}^{p}\left(1-\epsilon_{k} r_{k}\right)
$$

where

$$
\epsilon_{k}=1 \text { if } \theta_{k}=0, \quad \epsilon_{k}=-1 \text { if } \theta_{k}=1 .
$$

It will be useful to observe that if $x$ is given by (6) we can also write

$$
\lambda_{\theta k}^{(k)}=(1 / 2)\left(1-\epsilon_{k} r_{k}\right)=(1 / 2)\left(1-\phi_{k}(x) r_{k}\right)
$$

where $\left\{\phi_{k}(x)\right\}$ denotes the system of Rademacher's functions $(k=1,2, \cdots)$. Thus, with this notation, the inequality (8) is written

$$
F\left(x^{\prime}\right)-F(x)<\left(1 / 2^{p}\right) \prod_{k=1}^{p}\left(1-\phi_{k}(x) r_{k}\right) .
$$

We can now prove the following theorem.

THEOREM. The function $F(x)$ is purely singular when, and only when, the series $\sum r_{k}^{2}$ diverges. 
We shall make use of the following theorem, due to Zygmund( $\left.{ }^{2}\right)$ : for almost all $x$ we have

$$
\lim \inf \sum_{1}^{n}-r_{k} \phi_{k}(x)=-\infty
$$

if the series $\sum r_{k}^{2}$ diverges.

We deduce immediately from this result and from the inequality

$$
1-r_{k} \phi_{k}(x)<e^{-r_{k} \phi_{k}(x)}
$$

that for almost all $x$

$$
\liminf _{p=\infty} \prod_{k=1}^{p}\left(1-r_{k} \phi_{k}(x)\right)=0
$$

provided that $\sum r_{k}^{2}=\infty$.

The proof of the first part of our theorem is now immediate. Taking an $x$ belonging to the set $E$ (meas $E=1$ ) for which (9) holds, we have

$$
\left|F\left(x+\left(\epsilon_{p+1} / 2^{p+1}\right)\right)-F(x)\right|<\left(1 / 2^{p}\right) \prod_{k=1}^{p}\left(1-\phi_{k}(x) r_{k}\right),
$$

hence

$$
\lim \inf 2^{p+1}\left|F\left(x+\left(\epsilon_{p+1} / 2^{p+1}\right)\right)-F(x)\right|=0
$$

and if $F^{\prime}(x)$ exists it is equal to zero. The proof is completed as above.

To prove the second part of our theorem let us suppose that $\sum r_{k}^{2}<\infty$. We know by a classical theorem that in this case the series $\sum r_{k} \phi_{k}(x)$ converges in a set $\varepsilon$ of measure 1 . From this and from the hypothesis $\sum r_{\boldsymbol{k}}^{2}<\infty$ it is easy to deduce that the infinite product

$$
\prod_{1}^{\infty}\left(1-r_{k} \phi_{k}(x)\right)
$$

is convergent when $x$ belongs to $\varepsilon$. Fixing an $x$ belonging to $\varepsilon$, let us remark that $\epsilon_{p+1}$ having the same signification as above the dyadic developments of $x$ and $x+\epsilon_{p+1} / 2^{p+1}$ have all their digits equal except the digits of rank $p+1$.

From this it is easy to deduce, for example geometrically, that

$$
\begin{aligned}
\frac{1-\left|r_{p+1}\right|}{2} \lambda_{\theta_{1}}^{(1)} \cdots \lambda_{\theta_{p}}^{(p)} & <\left|F\left(x+\frac{\epsilon_{p+1}}{2^{p+1}}\right)-F(x)\right| \\
& <\frac{1+\left|r_{p+1}\right|}{2} \lambda_{\theta_{1}}^{(1)} \cdots \lambda_{\theta_{p}}^{(p)} .
\end{aligned}
$$

(2) Zygmund, On lacunary trigonometric series, Trans. Amer. Math. Soc. vol. 34 (1932) p. 435. The proof given there for lacunary trigonometric series is immediately applicable to Rademacher's functions. 
Now the first part of this inequality together with the convergence of the product (10) shows that

$$
\lim \inf 2^{p+1}\left|F\left(x+\frac{\epsilon_{p+1}}{2^{p+1}}\right)-F(x)\right|>0 .
$$

Hence, whenever $F^{\prime}(x)$ exists for $x \in \mathcal{E}, F^{\prime}(x)$ is not zero. Remembering that $F^{\prime}(x)$ exists and is finite almost everywhere, we have that $F^{\prime}(x) \neq 0$ almost everywhere, and thus $F(x)$ cannot be purely singular. This completes the proof of the theorem.

Modulus of continuity of $F(x)$. The argument is the same as before. If

$$
1 / 2^{p+1} \leqq x^{\prime}-x<1 / 2^{p},
$$

we have

$$
F\left(x^{\prime}\right)-F(x)<2 \prod_{k=1}^{p}\left(\frac{1+\left|r_{k}\right|}{2}\right) .
$$

Thus if $\omega(\delta)$ is the modulus of continuity, we have

$$
\omega(\delta)<4 \prod_{k=1}^{(|\log \delta| / \log 2)}\left(\frac{1+\left|r_{k}\right|}{2}\right) .
$$

Fourier-Stieltjes coefficients of $F(x / 2 \pi)$. There is no change in the argument used above for $f(x)$ to prove that if

$$
c_{n}=\int_{0}^{2 \pi} e^{n i x} d F\left(\frac{x}{2 \pi}\right)
$$

we have for approximate expression of $c_{n}$

$$
\sum \lambda_{\theta_{1}}^{(1)} \cdots \lambda_{\theta_{p}}^{(p)} e^{2 \pi n i\left(\theta_{1} / 2+\theta_{2} / 2^{2}+\cdots+\theta_{p} / 2^{p}\right)}
$$

the summation being extended to the $2^{p}$ combinations of the values 0 and 1 of the $\theta_{i}$. We get thus

$$
\begin{aligned}
c_{n} & =\prod_{k=1}^{\infty}\left[\lambda_{0}^{(k)}+\lambda_{1}^{(k)} e^{2 \pi n i / 2^{k}}\right] \\
& =e^{\pi n i} \prod_{k=1}^{\infty}\left[\lambda_{0}^{(k)} e^{-\pi n i / 2^{k}}+\lambda_{1}^{(k)} e^{\pi n i / 2^{k}}\right] \\
& =e^{\pi n i} \prod_{k=1}^{\infty}\left[\cos \left(\pi n / 2^{k}\right)+i r_{k} \sin \left(\pi n / 2^{k}\right)\right]
\end{aligned}
$$

and

$$
\left|c_{n}\right|^{2}=\prod_{k=1}^{\infty}\left[\cos ^{2}\left(\pi n / 2^{k}\right)+r_{k}^{2} \sin ^{2}\left(\pi n / 2^{k}\right)\right] .
$$


It is immediately seen-as in the case of $f(x)$-that if $r_{k}$ does not tend to zero, we have $\left|c_{n}\right| \neq o(1)$.

4. The Minkowski function ? $(x)$. This function was defined by Minkowski( $\left.{ }^{3}\right)$ for the purpose of establishing a one-one correspondence between the rational numbers of $(0,1)$ and the quadratic irrationals of $(0,1)$. The properties of the function have been recently investigated by Denjoy $\left({ }^{4}\right)$ who has proved that it is purely singular and given other important properties and generalizations of Minkowski's function.

We propose to give here some new indications about this function, concerning particularly its modulus of continuity and its Fourier-Stieltjes coefficients. For the sake of completeness we shall give the definition of the function and the proof of its singularity.

Definition of the function ? $(x)$. We define first

$$
?(0)=?(0 / 1)=0, \quad ?(1)=?(1 / 1)=1 .
$$

We next take the "mediant" $1 / 2=(0+1) /(1+1)$ of the two Farey fractions $0 / 1$ and $1 / 1$ and we define ? $(0+1 / 1+1)$ to be the arithmetic mean between $?(0)$ and ?(1), that is, $1 / 2$.

We define in the same way

$$
\begin{aligned}
& ?(1 / 3)=?\left(\frac{0+1}{1+2}\right)=\frac{?(0)+?(1 / 2)}{2}=1 / 4, \\
& ?(2 / 3)=?\left(\frac{1+1}{2+1}\right)=\frac{?(1 / 2)+?(1)}{2}=3 / 4 .
\end{aligned}
$$

Generally if, by this process, we have defined $?(p / q)$ and $?\left(p^{\prime} / q^{\prime}\right)$ for two consecutive irreducible fractions $p / q, p^{\prime} / q^{\prime}$, we define

$$
?\left(\frac{p+p^{\prime}}{q+q^{\prime}}\right)=\frac{?(p / q)+?\left(p^{\prime} / q^{\prime}\right)}{2} .
$$

At the $n$th stage the function is defined for $2^{n}+1$ values of $x$ and the ordinates corresponding to these values of $x$ are of the form $k / 2^{n}$ $\left(k=0,1,2, \cdots, 2^{n}\right)$. The definition of $?(x)$ for every $x$ follows by continuity.

Let now $x$ be a rational number put in the form of a finite continued fraction:

$$
x=\left(a_{0}, a_{1}, \cdots, a_{n}\right), \quad a_{0}=0 \quad(0 \leqq x \leqq 1) .
$$

Let $p_{0} / q_{0}, p_{1} / q_{1}, \cdots, p_{n} / q_{n}=x$ be the successive convergents $\left(p_{0} / q_{0}=0\right.$, $\left.p_{1} / q_{1}=1 / a_{1}, \cdots\right)$. Let us assume that at a certain stage (the $m$ th) of the

(3) H. Minkowski, Gesammelte Abhandlungen vol. 2 (1911) pp. 50-51.

(4) A. Denjoy, C. R. Acad. Sci. Paris vol. 194 (1932) pp. 44-46 and J. Math. Pures Appl. vol. 17 (1938) pp. 105-151. 
construction of $?(x)$ the fractions $p_{k-2} / q_{k-2}, p_{k-1} / q_{k-1}$ are consecutive. (This happens certainly for $p_{0} / q_{0}=0$ and $p_{1} / q_{1}=1 / a_{1}, 1 / a_{1}$ appearing as consecutive to $0 / 1$ at the $\left(a_{1}-1\right)$ th stage of the construction.) Let

By definition

$$
y_{k-2}=?\left(\frac{p_{k-2}}{q_{k-2}}\right), \quad y_{k-1}=?\left(\frac{p_{k-1}}{q_{k-1}}\right) .
$$

$$
?\left(\frac{p_{k-1}+p_{k-2}}{q_{k-1}+q_{k-2}}\right)=\frac{y_{k-1}+y_{k-2}}{2} .
$$

Now it is well known that $\left(p_{k-1}+p_{k-2}\right) /\left(q_{k-1}+q_{k-2}\right)$ is irreducible and thus at the next stage (the $(m+1)$ th) the fraction (irreducible) $\left(2 p_{k-1}+p_{k-2}\right)$ $/\left(2 q_{k-1}+q_{k-2}\right)$ will appear with

$$
?\left(\frac{2 p_{k-1}+p_{k-2}}{2 q_{k-1}+q_{k-2}}\right)=\frac{y_{k-1}+\left(y_{k-1}+y_{k-2}\right) / 2}{2} .
$$

Continuing in the same way we see that

$$
\begin{aligned}
?\left(\frac{p_{k}}{q_{k}}\right) & =?\left(\frac{a_{k} p_{k-1}+p_{k-2}}{a_{k} q_{k-1}+q_{k-2}}\right) \\
& =\frac{y_{k-1}}{2}+\frac{y_{k-1}}{2^{2}}+\cdots+\frac{y_{k-1}}{2^{a_{k}}}+\frac{y_{k-2}}{2^{a_{k}}},
\end{aligned}
$$

hence, if we put $?\left(p_{k} / q_{k}\right)=y_{k}$,

$$
y_{k}=\left(1-\left(1 / 2^{a_{k}}\right)\right) y_{k-1}+y_{k-2} / 2^{a_{k}}
$$

or

$$
y_{k}-y_{k-1}=-\left(1 / 2^{a_{k}}\right)\left(y_{k-1}-y_{k-2}\right) \text {. }
$$

Now $p_{k} / q_{k}$ when it appears is consecutive to $p_{k-1} / q_{k-1}$. Hence we can repeat the argument, and if $y_{n}=?\left(p_{n} / q_{n}\right)=?(x)$, we have

$$
y_{n}-y_{n-1}=\left(-1 / 2^{a_{n}}\right)\left(-1 / 2^{a_{n-1}}\right) \cdots\left(-1 / 2^{a_{2}}\right)\left(y_{1}-y_{0}\right) .
$$

Now $y_{0}=0, y_{1}=1 / 2^{a_{1}-1}$, hence

and thus

$$
y_{n}-y_{n-1}=(-1)^{n-1} \frac{1}{2^{\left(a_{1}+\cdots+a_{n}\right)-1}} .
$$

$$
y_{n}=\frac{1}{2^{a_{1}-1}}-\frac{1}{2^{\left(a_{1}+a_{2}\right)-1}}+\frac{1}{2^{\left(a_{1}+a_{2}+a_{2}\right)-1}}-\cdots+(-1)^{n-1} \frac{1}{2^{\left(a_{1}+\cdots+a_{n}\right)-1}} .
$$

Now by continuity we get the following result: if

$$
x=\left(0, a_{1}, a_{2}, \cdots, a_{n}, \cdots\right),
$$


we have

$$
?(x)=\frac{1}{2^{a_{1}-1}}-\frac{1}{2^{\left(a_{1}+a_{2}\right)-1}}+\cdots+(-1)^{n-1} \frac{1}{2^{\left(a_{1}+\cdots+a_{n}\right)-1}}+\cdots,
$$

and it is easy to see that if $x$ is rational, the two different developments of $x$ give the same ? $(x)$.

From this we deduce the more elementary properties of ? $(x)$, namely:

If $x$ is rational, ? $(x)$ is of the form $k / 2^{*}$ ( $k, s$ integers).

If $x$ is irrational, the dyadic development of ? $(x)$ is infinite.

If $x$ is a quadratic irrational $\left(0, a_{1}, a_{2}, \cdots\right)$ is periodic and thus ? $(x)$, being the difference of two periodic dyadic developments, is rational.

It is not difficult to see that the reciprocals of these results are true.

The fact that ? $(x)$ is strictly increasing is an immediate consequence of its construction.

Proof of the singularity of ? $(x)$. Let $x=\left(0, a_{1}, \cdots, a_{n}, \cdots\right)$. We know that for almost all $x \lim \sup a_{n}=\infty$. Let $N$ be the set of such numbers (meas. $N=1)$ and let us fix an $x$ belonging to $N$. Let $x=\left(0, a_{1}, \cdots, a_{n}, \cdots\right)$, let $?(x)=y$ and let

$$
r_{n}=p_{n} / q_{n}=\left(0, a_{1}, \cdots, a_{n}\right), \quad \rho_{n}=?\left(r_{n}\right),
$$

and let us write, as usual $a_{n+1}^{\prime}=\left(a_{n+1}, a_{n+2}, \cdots\right)$. We have

$$
x=\frac{a_{n+1}^{\prime} p_{n}+p_{n-1}}{a_{n+1}^{\prime} q_{n}+q_{n-1}}, \quad\left|x-\frac{p_{n}}{q_{n}}\right|=\frac{1}{\left(a_{n+1}^{\prime} q_{n}+q_{n-1}\right) q_{n}},
$$

and thus

$$
\frac{1}{\left(a_{n+1}+2\right) q_{n}^{2}}<\left|x-\frac{p_{n}}{q_{n}}\right|<\frac{1}{a_{n+1} q_{n}^{2}}
$$

and

$$
y-\rho_{n}=(-1)^{n}\left[\frac{1}{2^{\left(a_{1}+\cdots+a_{n+1}\right)-1}}-\frac{1}{2^{\left(a_{1}+\cdots+a_{n+2}\right)-1}}+\cdots\right]
$$

which gives

$$
\frac{1}{2^{a_{1}+\cdots+a_{n+1}}}<\left|y-\rho_{n}\right|<\frac{1}{2^{\left(a_{1}+\cdots+a_{n+1}\right)-1}} .
$$

Hence, we have

and

$$
\delta_{n}=\left|\frac{y-\rho_{n}}{x-r_{n}}\right|<\frac{2\left(a_{n+1}+2\right) q_{n}^{2}}{2^{a_{1}+\cdots+a_{n+1}}}
$$




$$
\delta_{n-1}=\left|\frac{y-\rho_{n-1}}{x-r_{n-1}}\right|>\frac{a_{n} q_{n-1}^{2}}{2^{a_{1}+\cdots+a_{n}}} .
$$

Consequently,

$$
\begin{aligned}
\frac{\delta_{n}}{\delta_{n-1}} & <2 \frac{1}{2^{a_{n+1}}}\left(\frac{a_{n+1}+2}{a_{n}}\right)\left(\frac{q_{n}}{q_{n-1}}\right)^{2} \\
& <2 \frac{1}{2^{a_{n+1}}}\left(\frac{a_{n+1}+2}{a_{n}}\right)\left(a_{n}+1\right)^{2}<C \frac{a_{n} a_{n+1}}{2^{a_{n+1}}},
\end{aligned}
$$

$C$ being an absolute constant.

Now we can certainly find an infinite subsequence $\left\{a_{n_{k}}\right\}$ of the $\left\{a_{n}\right\}$ such that $a_{n_{k}}<a_{n_{k}+1}$ and $a_{n_{k}} \rightarrow \infty$, hence

$$
\lim \inf \delta_{n} / \delta_{n-1}=0 .
$$

Now if $d y / d x$ exists, is finite, and is different from zero at the point $x$, $\delta_{n} / \delta_{n-1}$ must tend to 1 . Hence, at any point $x \in N, d y / d x$ cannot exist, be finite, and be different from zero. But $d y / d x$ exists and has a finite value almost everywhere. Then the only possible conclusion is $d y / d x=0$ almost everywhere, which proves the singularity of the function.

Modulus of continuity of ? $(x)$. We need the following result on continued fractions, which to our knowledge has not been stated:

Lemma. Let $p_{n} / q_{n}=\left(0, a_{1}, a_{2}, \cdots, a_{n}\right)$. Let $\theta$ be the Fibonacci number $(1 / 2)\left(5^{1 / 2}+1\right)$. We have the inequality $q_{n}<\theta^{a_{1}+a_{2}}+\cdots+a_{n}$.

We shall prove this lemma by induction. We have $q_{1}=a_{1}<\theta^{a_{1}}$ for it is easily seen that $m<\theta^{m}$ for every positive integer $m$. We have also $q_{0}=1=\theta^{0}$. And we have generally $q_{k}=a_{k} q_{k-1}+q_{k-2}(k=2,3, \cdots, n)$. If supposing the lemma true for $n=k-2$ and $n=k-1$ we prove that it is true for $n=k$, we will have proved the result as stated. Let

$$
q_{k-1}<\theta^{a_{1}+\cdots+a_{k-1}}, \quad q_{k-2} \leqq \theta^{a_{1}+\cdots+a_{k-2}} .
$$

It is sufficient to prove that

$$
a_{k} \theta^{a_{1}+\cdots+a_{k-1}}+\theta^{a_{1}+\cdots+a_{k-2}} \leqq \theta^{a_{1}+\cdots+a_{k}},
$$

that is, $a_{k} \theta^{a_{k-1}}+1 \leqq \theta^{a_{k-1}+a_{k}}$ or $a_{k}+1 / \theta^{a_{k-1}} \leqq \theta^{a_{k}}$. Hence it is sufficient to prove that $a_{k}+1 / \theta \leqq \theta^{a_{k}}$. Now for $a_{k}=1$ we have the equality $1+1 / \theta=\theta$ and it is easy to see that $2+1 / \theta=\theta^{2}$ and that the function $\theta^{x}-x$ increases when $x \geqq 2$. Hence the lemma is proved. (It is easy to see, by considering the number $(0,1,1,1, \cdots)$ that this result is the best possible of its kind, in order of magnitude.)

We can now proceed to determine the modulus of continuity of ?(x). In the definition of the function by successive approximations, we start from the 
Farey fractions $0 / 1$ and $1 / 1$ and in a first operation we introduce the mediant $1 / 2$, in a second operation the two mediants $1 / 3$ and $2 / 3$, in a third operation, four mediants, and so on. In the pth operation we introduce $2^{p-1}$ mediants and we get a sequence of fractions containing

$$
2+\left(1+2+\cdots+2^{p-1}\right)=2^{p}+1
$$

fractions, which we can call the Minkowski sequence of order $p$ and denote by $\mathfrak{M}_{p}$. To the sequence $\mathfrak{M}_{p}$ corresponds, by the transformation $y=$ ? $(x)$, the sequence of numbers $k / 2^{p}\left(k=0,1,2, \cdots, 2^{p}\right)$. The formula (11) giving the value of ? $(x)$ shows that the fractions belonging to $\mathfrak{M}_{p}$ are those which, when written in the form $\left(0, a_{1}, a_{2}, \cdots, a_{n}\right)$, are such that $\sum_{1}^{n} a_{\nu}$ does not exceed $p+1$. Hence, by the lemma, if $\alpha / \beta$ belongs to $\mathfrak{M}_{p}$, we have $\beta<\theta^{p+1}$, and this order of magnitude is actually attained for the fraction $(0,1,1, \ldots, 1)$ where 1 is repeated $p+1$ times. Now it is immediately seen, by induction, that if $\alpha / \beta$, $\alpha^{\prime} / \beta^{\prime}$ are two consecutive fractions of $\mathfrak{M}_{p}$, we have $\left|\beta^{\prime} \alpha-\beta \alpha^{\prime}\right|=1$ and thus the distance between two consecutive fractions of $\mathfrak{M}_{p}$ is greater than $1 / \theta^{2 p+2}$.

Let now $x, x^{\prime}$ be two irrational points of $(0,1), y=?(x), y^{\prime}=?\left(x^{\prime}\right)$. At a certain stage of the dissection one fraction $x_{0}$ appears for the first time in $\left(x, x^{\prime}\right)$. Let us continue the dissection until one fraction appears for the first time in $\left(x, x_{0}\right)$ or in $\left(x_{0}, x^{\prime}\right)$ or in both intervals. Let this stage of the dissection be the $p$ th, then we have $x^{\prime}-x>1 / \theta^{2 p+2}$ and $y^{\prime}-y<4 / 2^{p}$. Hence,

$$
(2 p+2) \log \theta>\log \left|\frac{1}{x^{\prime}-x}\right|, \quad(p-2) \log 2<\log \left|\frac{1}{y^{\prime}-y}\right|
$$

which proves that

$$
\left|y^{\prime}-y\right|<\left|x^{\prime}-x\right|^{2^{-1} \log 2 / \log \theta} \text {. }
$$

$C$ being an absolute constant, and this relation being true for every couple of irrationals $x, x^{\prime}$ is also valid for $x$ or $x^{\prime}$ or both rational. Hence: the function $?(x)$ satisfies a Lipschitz condition of order $\alpha=(1 / 2) \log 2 / \log \theta$ where $\theta$ is the libonacci number $(1 / 2)\left(5^{1 / 2}+1\right)$.

We shall now prove that $\alpha$ is the best possible exponent for the Lipschitz condition of $?(x)$ and that it cannot be improved.

Let us consider, in fact, the number

$$
\chi=(0,1,1, \cdots)=\left(5^{1 / 2}-1\right) / 2=1 / \theta .
$$

The corresponding value of the function is

$$
\eta=?(\chi)=1-1 / 2+1 / 2^{2}-\cdots=2 / 3 .
$$

Let $p_{n} / q_{n}$ be the successive convergents of $\chi$. It is well known that

$$
q_{n}=\left(1 / 5^{1 / 2}\right)\left[\theta^{n+1}-(-1)^{n+1}\left(1 / \theta^{n+1}\right)\right], \quad p_{n}=q_{n-1} .
$$


Now

$$
\delta=\left|\chi-p_{n} / q_{n}\right|<1 / q_{n}^{2}
$$

which is of the same order as $1 / \theta^{2 n}$, whereas

$$
\eta-?\left(p_{n} / q_{n}\right)=(-1)^{n}\left(1 / 2^{n}\right)+(-1)^{n+1}\left(1 / 2^{n+1}\right)+\cdots
$$

is of order $1 / 2^{n}$, that is, of order $\delta^{2^{-1}} \log 2 / \log \theta$, which proves that the number $\alpha$ of our Lipschitz condition is the best possible one.

Fourier-Stieltjes coefficients of ? $(x / 2 \pi)$. Let

$$
c_{n}=\int_{0}^{2 \pi} e^{n i x} d ?(x / 2 \pi)
$$

It is immediately seen that

$$
c_{n}=\lim _{p=\infty}\left[\frac{1}{2^{p}} \sum_{\rho} \mathfrak{M}_{p} e^{2 \pi n i \rho}\right]
$$

where the summation is extended to all fractions $\rho$ belonging to $\mathfrak{M}_{p}$.

It does not seem to be known whether $c_{n}$ tends to zero for $n=\infty$. If we confine ourselves to the behavior of $c_{n}$ "in the average," we get the following result. It is well known by a theorem of Wiener $\left(^{5}\right)$ that

$$
\left|c_{1}\right|^{2}+\left|c_{2}\right|^{2}+\cdots+\left|c_{n}\right|^{2}<A n \omega(1 / n)
$$

$\omega(\delta)$ being the modulus of continuity of the function and $A$ an absolute constant. Hence, by our result on the modulus of continuity of ? $(x)$ we have

$$
\left|c_{1}\right|^{2}+\left|c_{2}\right|^{2}+\cdots+\left|c_{n}\right|^{2}=O\left(n^{1-2^{-1} \log 2 / \log \theta}\right)
$$

and, by Schwarz's inequality

$$
\left|c_{1}\right|+\left|c_{2}\right|+\cdots+\left|c_{n}\right|=O\left(n^{14^{-1} \log 2 / \log \theta}\right) .
$$

(b) See, for example, Zygmund, Trigonometrical series, p. 221.

Harvard UNIVERSITy,

Cambridge, Mass.

Massachusetts Institute of Technology,

Cambridge, Mass. 